\title{
Prácticas de alimentación del lactante y del niño pequeño en tres instituciones de salud en el Caribe colombiano, bajo la estrategia "Instituciones amigas de la mujer y la infancia integral"*
}

\author{
Feeding practices of the infant and child, in three health institutions \\ in the Colombian Caribbean
}
Práticas de alimentação do lactante y del niño pequeño en tres instituciones de salud en el Caribe colombiano, bajo la estrategia "Instituciones amigas de la mujer y la infancia integral "

\begin{abstract}
Nancy Haydeé Millán Echevarría; Paula Andrea Castro Prieto2; Sonia Cristina Fernández Fernández³; Gina Paola Arocha Zuluaga ${ }^{4}$

1 Magíster en Salud Intercultural, con Mención en Salud Sexual y Reproductiva. Consultora independiente en salud y nutrición. Colombia.nanmillane@gmail.com. oRcID: https://orcid.org/0000-0001-7940-673X

2 Magíster en Salud Pública. Fundación Santa Fe de Bogotá. Colombia. paula.castro@fsfb.org.co. oRCID: https://orcid.org/0000-00031333-7983

3 Máster en Trastornos del Comportamiento Alimentario y Obesidad. Colsanitas. Colombia. kriztina9@hotmail.com oRcid: https://orcid. org/0000-0001-6587-3273

4 Magíster en Desarrollo Educativo y Social. Consultora independiente en salud y nutrición materno infantil. Bogotá, Colombia. ginaarocha@gmail.com. oRCID: https://orcid.org/0000-0002-4566-1406
\end{abstract}

Recibido: 17/06/2020. Aprobado: 04/04/2021. Publicado: 30/04/2021

Millán NH, Castro PA, Fernández SC, Arocha GP. Prácticas de alimentación del lactante y del niño pequeño en tres instituciones de salud en el Caribe colombiano bajo la estrategia "Instituciones amigas de la mujer y la infancia integral". Rev. Fac. Nac. Salud Pública. 2021;39(2):e343224. Dor: https://doi.org/10.17533/udea.rfnsp.e343224

\section{Resumen}

Objetivo: Describir las prácticas de alimentación de niños lactantes y de niños pequeños atendidos en instituciones de tres municipios del Caribe colombiano, incluidos en el proceso de implementación de la estrategia "Instituciones amigas de la mujer y la infancia integral" de la Unicef. Metodología: Estudio cualitativo enmarcado en la etnografía, llevado a cabo mediante entrevistas semiestructuradas, cartografía social y grupos focales, dirigido a madres beneficiarias de instituciones

* Este trabajo parte de un avance del estudio sobre los determinantes sociodemográficos de la salud asociados con el estado nutricional en Colombia, elaborado por la estudiante Paula Castro, en el marco del programa de Doctorado en Demografía, del Centre d'Estudis Demogràfics, Universitat Autònoma de Barcelona (España), y que ha sido ampliado en concurso con los demás autores del presente artículo. 
de salud priorizadas. Resultados: Las madres reconocieron los beneficios nutricionales de la lactancia materna, y en dos de los territorios, los beneficios emocionales. Además, presentan apropiación en lo relacionado con la alimentación perceptiva. No obstante, no cumplen con el periodo de exclusividad de la lactancia, refieren algunas creencias populares acerca de la misma y se observa un inicio temprano de la alimentación complementaria. También reconocieron la importancia de las redes de apoyo familiar y social, como, por ejemplo, del Instituto Colombiano de Bienestar Familiar. Conclusión: Esta investigación permitió reconocer factores que favorecen y limitan las adecuadas prácticas de alimentación del lactante y del niño pequeño, en madres gestantes y lactantes y su red de apoyo, las cuales viven en condiciones de vulnerabilidad social y económica, y son atendidas en tres instituciones de salud del Caribe colombiano que se encuentran en proceso de implementación de la estrategia enunciada. Lo anterior brinda elementos para la formulación de procesos educativos en salud para la población materno infantil, tanto en la población objetivo como en instituciones que se encuentren en proceso de implementación de dicha estrategia, y de requerimientos políticos acerca de la salud materno infantil.

-Palabras clave: alimentación complementaria, cuidado del niño, "Instituciones amigas de la mujer y la infancia integral" (Unicef), lactancia materna, nutrición de lactante, redes comunitarias.

\section{Abstract}

Objective: Describe the infant and young child feeding practices of children cared for in institutions of the Colombian Caribbean, includes in implementing the baby friendly hospital strategy. Methods: Qualitative study framed in ethnography through semi-structured interviews, aimed at beneficiary mothers of prioritized health institutions. Results: The mothers recognized the nutrtitionals benefits of breastfeeding, in two territories also the emotional benefits. They also show appropriation in relation to perceptual feeding. However, they do not comply with the exclusive period of breastfeeding, they refer to some beliefs around it, an early start of complementary feeding is observed. In turn, the mothers recognized the importance of family and social support networks, such as the Instituto Colombiano de Bienestar Familiar. Conclusion: This research made it possible to recognize factors that favor and limit the adequate feeding practices of infants and young children, in pregnant and lactating mothers and their support network, who live in conditions of social and economic vulnerability and are cared for in three health institutions of the Colombian Caribbean that are in the process of implementing the babyfriendly hospitals initiative. The foregoing provides elements for the formulation of educational processes in health in the maternal and child population, both in the target population and in institutions that are in the process of implementing the baby-friendly hospitals initiative and policies to maternal and child health.

---------Keywords: complementary feeding, childcare, baby friendly hospital strategy, breastfeeding, infant nutrition, community networks.

\section{Resumo}

Objetivo: Descrever as práticas alimentares de lactentes e crianças atendidas em instituições de três municípios do Caribe colombiano, incluídas no processo de implementação da estratégia do Unicef "Instituições Amigas da Mulher e Infância Integral”. Métodos: Estudo qualitativo enquadrado na etnografia, realizado por meio de entrevistas semiestruturadas, cartografia social e grupos focais, dirigido a mães beneficiárias de instituições de saúde priorizadas. Resultados: As mães reconheceram os benefícios nutricionais da amamentação e, em dois dos territórios, os benefícios emocionais. Além disso, apresentam apropriação em relação à alimentação perceptiva. Porém, não cumprem o período exclusivo de aleitamento materno, referem-se a algumas crenças populares a respeito, e observa-se o início precoce da alimentação complementar. Também reconheceram a importância das redes de apoio familiar e social, como o Instituto Colombiano de Bem-Estar
Familiar. Conclusão: Esta pesquisa permitiu reconhecer fatores que favorecem e limitam as práticas alimentares adequadas de lactentes e crianças pequenas, em gestantes e lactantes e sua rede de apoio, que vivem em condições de vulnerabilidade social e econômica, sendo atendidas em três. Instituições de saúde do Caribe colombiano que estão em processo de implementação da estratégia declarada. $\mathrm{O}$ exposto fornece elementos para a formulação de processos educativos em saúde para a população materno-infantil, tanto na população-alvo como nas instituições que estão em processo de implementação dessa estratégia, e de requisitos políticos relativos à saúde materno-infantil.

---------Palavras-chave: alimentação complementar, cuidado infantil, Iniciativa Hospital Amigo da Criança, lactância Materna, nutrição infantil, Redes Comunitárias. 


\section{Introducción}

La estrategia "Instituciones amigas de la mujer y la infancia integral" (IAMII) fue propuesta a Colombia por el Fondo de las Naciones Unidas para la Infancia (Unicef), con el propósito de que las instituciones de salud mejoren prácticas de atención en salud y nutrición materna e infantil [1, p. 10]. La estrategia promueve que las instituciones que prestan servicios de atención a mujeres, gestantes, madres, niñas, niños y adolescentes, desarrollen una metodología que permita mejorar las prácticas de salud y nutrición materna e infantil, a partir de la puesta en marcha de sus diez pasos.

Los diez pasos de la IAMII son: 1) voluntad política institucional, 2) armonización conceptual y práctica del personal de salud en torno a la salud y nutrición materna e infantil, 3) empoderamiento de las mujeres y sus familias para el cuidado, 4) mejores condiciones para el nacimiento, 5) ayuda efectiva para iniciar el cuidado del recién nacido y de la puérpera, 6) promoción, protección y apoyo efectivo de la lactancia materna, 7) favorecer el alojamiento y el acompañamiento, 8) seguimiento sistemático al crecimiento y desarrollo de los niños y niñas, 9) la institución de salud como entorno protector y un espacio amigable, y 10) cuidado de la salud y nutrición materna e infantil más allá de la institución de salud [1, pp. 8-9]. Este último paso involucra "Disponer de mecanismos y estrategias de apoyo institucional y comunitario que favorezcan la continuidad de las acciones más allá de los servicios institucionales, con el fin de favorecer la salud y la nutrición materna e infantil" [1, p. 9].

A nivel internacional, esta estrategia también es conocida como "Hospitales amigos de los niños", los cuales han reportado avances en los indicadores de salud y nutrición materno infantil. En Los Ángeles, Estados Unidos de América, un estudio determinó que los hospitales en proceso de certificación o certificados con la estrategia se asociaron significativamente con la probabilidad de que las madres amamantaran de forma exclusiva entre 1 y 3 meses [2]. En el mismo país, se evaluó el impacto de los hospitales de los niños sobre el inicio temprano de la lactancia materna, encontrando que el inicio y la exclusividad de la lactancia materna tiende a ser exitosa cuando las madres tienen mayor exposición a las prácticas amigables con los bebés; sin embargo, se hallan deficiencias para realizar el seguimiento a las madres por parte de la institución y en la consolidación de grupos de apoyo de la lactancia [3].

En Brasil, un análisis de la evaluación interna y externa del cumplimiento de la estrategia, en hospitales certificados como "amigos de los niños", evidenció que las instituciones de salud son espacios protectores y amigables con la lactancia, y los grupos de apoyo se encontraban en un $95 \%$ de cumplimiento, derivado de una red de la lactancia materna brasileña [4].

En Colombia, un estudio del 2002, efectuado en hospitales del Valle del Cauca, identificó que la implementación de la estrategia era baja. En lo concerniente al paso 10, sugirió la vinculación de la institución con los grupos de apoyo comunitarios ya establecidos, para de este modo fortalecer la práctica de la lactancia materna [5]. De igual forma, en Cartagena, una investigación estableció el grado de percepción de implementación de la estrategia en ocho instituciones de la ciudad, encontrando que la conformación de grupos de apoyo presentó un grado bajo de implementación [6].

La IAMII, al ser una estrategia que fortalece el mejoramiento de las prácticas de alimentación infantil, fomenta la lactancia materna exclusiva hasta los 6 meses y con alimentación complementaria hasta los 2 años o más, por lo cual estos indicadores resultan trazadores. De acuerdo con la Organización Mundial de la Salud (OMS), en América del Norte solo el $26 \%$ de los lactantes menores de 6 meses reciben exclusivamente leche materna [7]. En México, este indicador alcanzó el 28,6 \% [8]; mientras que en Perú, Bolivia y Argentina, las cifras ascienden a $69,8,60$ y $53 \%$, respectivamente [9-11].

A su vez, la alimentación complementaria en países como México es iniciada antes de los 6 meses, predominantemente con frutas, jugos industrializados, refrescos y frituras [12]. En Perú, la introducción de las preparaciones y de la leche no materna en la dieta ocurrió a los 6 meses, con excepción de aguas e infusiones, que comenzó a los 5 meses [13]. Para Argentina, se reportó que el $33,5 \%$ de los menores de 6 meses recibió alimentos sólidos [14]. De igual forma, un estudio en los municipios de Sacaca y San Pedro de Buena Vista, del departamento de Potosí en los Andes de Bolivia, estableció que el 39 $\%$ de los niños iniciaron la alimentación complementaria en los 3 primeros meses de vida (n: 400) [15].

En Colombia, la Encuesta Nacional de Situación Nutricional 2015 encontró que tan solo el 36,1 \% de los niños menores de 6 meses recibieron de manera exclusiva leche materna, lo cual reporta un descenso, puesto que para 2010 este indicador fue de $42,8 \%$, sumado a que el 3,3\% de los menores de 6 meses recibió, además de leche materna, otros líquidos no lácteos. Cabe anotar que la lactancia materna exclusiva en la región Caribe alcanzó el 20,5 \%. En cuanto a alimentación complementaria, a nivel nacional se observó que el $64 \%$ de los niños entre 6 y 8 meses presentaron una introducción apropiada de alimentos sólidos, semisólidos y blandos. Por su parte, el indicador de dieta mínima aceptable, que analiza la diversidad dietaria y la frecuencia de alimentación en los niños de 6 a 23 meses en niños amamantados y no amamantados, fue de 67,4 y $76,5 \%$, respectivamente [16]. 
Por el panorama descrito, la Fundación Santa Fe de Bogotá (FSFB), la Fundación Éxito (FE) y el Ministerio de Salud y Protección Social (MSPS), entes territoriales de salud de Cartagena, Barranquilla y Sincelejo, e instituciones de salud interesadas en la IAMII, establecieron una alianza para fortalecer los pasos 2 y 10 de la estrategia, por medio de un proceso que incluye cualificación en consejería en alimentación del lactante (niños menores de 6 meses) y del niño pequeño (niños entre 6 meses y 3 años), ofrecido a profesionales de la salud adscritos a instituciones en salud; asistencia técnica para la implementación de la estrategia, y la consolidación de redes de apoyo comunitario para la atención materno infantil, lo cual incide en la toma de decisiones informadas por parte de gestantes, lactantes y su red de apoyo, en lo relacionado con la lactancia materna y la alimentación complementaria.

Por lo anterior, el objetivo de la investigación que aquí se reporta es describir las prácticas de alimentación de lactantes y niños pequeños - es decir, lactancia materna y alimentación complementaria de niños - atendidos en instituciones de tres municipios del Caribe colombiano y que desplegaron acciones establecidas en la alianza institucional mencionada anteriormente, durante el año 2019, en el proceso de implementación de la estrategia IAMII.

Las tres instituciones fueron: el Camino Bosque de María, en Barranquilla; la Clínica General del Caribe, en Cartagena, y la Institución Prestadora de Salud (IPS) San Luis, en Sincelejo.

Cabe anotar que el MSPs fue el interlocutor en primer momento con los entes territoriales e instituciones de salud, y quien asumió el rol y el despliegue de la intervención fue la FSFB.

Hay que aclarar que esta intervención constituye un proyecto piloto, que aportó lecciones y orientaciones para el estudio cofinanciado por el Ministerio de Ciencia, Tecnología e Innovación, denominado "Efectividad de una intervención en salud pública, para la prevención del retraso en talla de la población menor de un año, de un área urbana del Distrito Especial de Barranquilla", que fue aprobado por el Comité de Ética de la Fundación Santa Fe de Bogotá (código CCEI-10734-2019).

\section{Metodología}

Estudio cualitativo, enmarcado en la etnografía, la cual tiende a desarrollar conceptos y a comprender las acciones humanas desde un punto de vista interno, esto es, desde las personas y los mismos actores sociales que les dan sentido y explicación a sus conceptos y a sus acciones humanas. A su vez, la etnografía permite registrar la forma en que las personas perciben las situaciones, lo que implica analizar la representación social, es decir, la valoración y las actitudes compartidas por los sujetos [17,18]. Además, la etnografía posibiita enfatizar en la exploración de la naturaleza particular de los fenómenos sociales, al tener una tendencia a trabajar primariamente con datos "inestructurados" [17].

Las herramientas aplicadas fueron (véase Tabla 1):

- Entrevista semiestructurada. Tiene como propósito conocer en detalle lo que piensa o siente una persona con respecto a un tema o una situación particular [19], permitiendo que los mismos actores sociales proporcionen los datos relativos a sus conductas, opiniones, deseos, actitudes, expectativas, lo cual es muy difícil de obtener por otros medios [20]. Esta fue aplicada a madres gestantes y lactantes de niños menores de 2 años, beneficiarias del proceso, atendidas por parte de las instituciones focalizadas.

- Cartografia social. Es una propuesta que permite aproximarse al territorio y construir un conocimiento integral de este, empleando instrumentos técnicos y vivenciales [21]. Se planteó un ejercicio de cartografía social para identificar redes de apoyo, el cual solo fue aplicado a madres gestantes y lactantes atendidas en la Clínica General del Caribe.

- Grupos focales. Constituye un tipo de entrevista grupal, que favorece el intercambio de opiniones y que muestra el grado de variedad de los puntos de vista que existen sobre un mismo tema [22]. Solo fue posible con madres gestantes y lactantes atendidas en la Clínica General del Caribe.

Tabla 1 Herramientas aplicadas en el componente cualitativo, por municipio.

\begin{tabular}{lccc}
\hline $\begin{array}{l}\text { Instrumento/ herramienta } \\
\text { metodológica }\end{array}$ & Cartagena & Barranquilla & Sincelejo \\
\hline Entrevista a mujeres & 8 & 9 & 11 \\
Grupos focales a usuarias & 1 (compuesto por 10 gestantes/lactantes) & $\begin{array}{c}\text { No se logró, por dificultades logísticas y de } \\
\text { convocatoria }\end{array}$ \\
Cartografía & 1 (compuesto por 10 gestantes/lactantes) & \\
Total de participantes & 38 gestantes/lactantes & \\
\hline
\end{tabular}




\section{Población estudio}

Para el desarrollo de esta investigación, se seleccionaron madres gestantes y lactantes atendidas en el Camino Bosque de María, en Barranquilla; la Clínica General del Caribe, en Cartagena, y la IPs San Luis, en Sincelejo.

Para la selección de gestantes y lactantes, los profesionales de la salud y líderes comunitarios, con la aprobación de los comités científicos y directivos de las tres instituciones de salud en Sincelejo (acta del 8 y el 9 de abril de 2019), Cartagena (acta del 15 de marzo de 2019) y en Barranquilla (acta del 13 de marzo de 2019), informaron a las gestantes y lactantes de niños menores de 2 años atendidas en los centros de salud priorizados, sobre esta investigación. Quienes deseaban participar, fueron inclui- das en el análisis. Esta primera etapa de convocatoria se ejecutó entre diciembre de 2019 y enero de 2020.

La segunda etapa, enfocada en la recolección de datos, fue llevada a cabo entre enero y febrero de 2020, por dos profesionales de salud con experiencia en análisis cualitativo. Los sitios empleados para esta actividad fueron los centros de salud y los salones sociales reconocidos por la comunidad. En total se contó con la participación de 38 gestantes o lactantes.

En cuanto a las variables propuestas, se enmarcaron en la práctica de la lactancia materna, la alimentación complementaria y las redes sociales que fortalecen el ejercicio de maternidad y cuidado de los niños (véase Tabla 2).

Tabla 2. Variables propuestas en la fase construcción de instrumentos metodológicos, componente cualitativo

\begin{tabular}{ll}
\hline \multicolumn{1}{c}{ Temática orientadora } & Variable \\
\hline \multirow{2}{*}{ Lactancia materna } & Exclusividad y beneficios \\
\hline & Prácticas y creencias \\
Alimentación complementaria & Alimentos consumidos por los niños \\
& Alimentos saludables \\
\hline Redes sociales que fortalecen el ejercicio de maternidad y cuidado de los niños & Alimentos no saludables \\
\hline
\end{tabular}

\section{Análisis de datos}

Para el análisis de los datos obtenidos mediante los instrumentos cualitativos, se realizó la transcripción de las entrevistas y de los aportes de las participantes en el grupo focal y la cartografía social adelantada en Cartagena. Posteriormente, se codificaron las transcripciones con ayuda del software Atlas-ti versión 8 (licencia de Salud Poblacional de la FSFB) y se formaron categorías a partir del número de códigos resultantes, para su interpretación, teorización y la construcción de una red semántica (triangulación de la información).

Dentro del marco de una investigación cualitativa, la triangulación comprende el uso de varias estrategias, al estudiar un mismo fenómeno, por ejemplo, mediante el uso de varios métodos (entrevistas individuales, grupos focales o talleres investigativos) o de diferentes fuentes de información. La triangulación permite que se elabore una perspectiva más amplia en cuanto a la interpretación del fenómeno en cuestión, porque señala su complejidad, y esto, a su vez, enriquece el estudio y brinda la

$\doteqdot$ Mediante las actas ya enunciadas en el apartado "Población estudio" oportunidad de que se realicen nuevos planteamientos. La triangulación ofrece la posibilidad de poder visualizar una situación o problema desde diferentes ángulos y de esta manera aumentar la validez y la consistencia de los hallazgos.

\section{Aspectos éticos}

De acuerdo con la Resolución 008430 de 1993 [23], esta investigación se considera de riesgo mínimo, puesto que es un estudio que empleó el registro de datos de prácticas rutinarias.

Para el desarrollo de esta investigación se contó con el aval de los comités científicos y directivos de los centros de Sincelejo, Cartagena y en Barranquilla. ${ }^{\dagger}$ Por su parte, las madres que participaron de las entrevistas y en el grupo focal firmaron y grabaron la autorización de participar en este análisis y de emplear imágenes e información con fines de investigación.

La información recolectada fue anonimizada y se encuentra resguardada por el equipo de investigación de la FSFB. 


\section{Resultados}

Es de aclarar que aproximadamente el $60 \%$ de las entrevistadas viven en condiciones socioeconómicas de pobreza y vulnerabilidad, y manifestaron pertenecer al régimen subsidiado de salud. ${ }^{*}$

A continuación se presentan los resultados, respondiendo a las tres temáticas orientadoras propuestas en la metodología: lactancia materna, alimentación complementaria y redes sociales que fortalecen el ejercicio de maternidad y cuidado de los niños.

\section{Lactancia materna}

Se identificó que aproximadamente el $80 \%$ de las mujeres gestantes y lactantes entrevistadas han introducido algún alimento antes de los 6 meses; inclusive, en sus relatos se observa un inicio a muy temprana edad (menos del mes). Algunas lo hacen por indicaciones del personal de salud de la institución donde tuvieron el parto. Adicionalmente, reciben la presión social y familiar, para que se introduzcan otros alimentos a temprana edad.

[...] le estoy dando fórmula. De 0 a 3 meses [...] para ayudarme, porque él llora mucho, él come mucho. Entonces, yo le doy mi pecho y queda como vacío y empieza a llorar, y se alza la cabeza. Entonces yo, como lloraba y lloraba, yo veía que a él el pecho, como que no lo estaba llenando; entonces, yo le doy el tetero y él se duerme más o menos una hora, dos horas, y se vuelve a parar, y otra vez la teta y después el tetero (Entrevista madre lactante E, Cartagena, 2020).

Frente a los beneficios de la leche materna, en Cartagena, la mayoría de las mujeres reconoce aquellos relacionados con el aporte nutricional, mientras que, en Barranquilla y Sincelejo, además mencionaron los beneficios de vínculo entre madre e hijo, quienes refirieron haber adquirido estos conocimientos en la institución de salud, y especialmente donde les atendieron el parto.

En estos dos últimos municipios, las madres mencionaron abiertamente los sentimientos de miedo, temor y alegría que les produce amamantar, mas no así en Cartagena, en gran medida porque las mujeres tenían dificultad para expresar sus sentimientos y emociones. Sin embargo, a pesar de reconocer los beneficios, aducen la necesidad de introducir otros alimentos (leches de tarro, aguas, u otros alimentos), porque los niños o las niñas "manifiestan" quedar con hambre, debido a la percepción que las madres tienen de que no producen suficiente leche para cubrir las necesidades del bebé, entre otras creencias.
Que el bebé se fortalece más, es más sano, y para nosotros, evitamos un cáncer de mamas [...] Que es preferible darle leche materna que cualquier leche de fórmula (Madre lactante N, Barranquilla, 2020).

Yo me siento alegre, porque siempre he sabido que la lactancia materna es la mejor; y aquí hay muchas mujeres, que conozco casos, que no pueden darle pecho a sus bebés. Y ya que yo sí se lo puedo dar, pues a mí me alegra mucho eso (Madre lactante G, Barranquilla, 2020).

Y también la charla esa que me ayudó bastante para informarme y esas cosas; pero, de todas maneras, como yo le digo, soy poca lechera; entonces, opté por comprarle los potes de leche (Madre lactante Y, Barranquilla, 2020).

En Sincelejo se encontraron dos casos de mujeres lactantes que garantizaron la práctica de la lactancia materna exclusiva, a pesar de las dificultades y la presión social para abandonarla, situación que acentuaban sus temores e inseguridades.

Llama la atención que, en Sincelejo, varias mujeres mencionaron claramente el acompañamiento educativo que tuvieron por parte de la entidad de salud en el curso de preparación para la maternidad y la paternidad.

[...] me dijeron la importancia de la lactancia materna; sabemos que el calostro es lo importante, porque es un método de defensa para el bebé, porque es lo que le va a ayudar para que el organismo como tal, el sistema lo ayuda como, viene siendo como si fuera el primer purgantico del bebé [...] que es lo que lo va a ayudar como tal. O sea, no hay necesidad de darle agua, porque al igual, la leche materna contiene todo (Madre lactante Y, Sincelejo, 2020).

Por su parte, los mitos hallados en torno a la práctica de la leche materna se relacionan en la Tabla 3.

\section{Alimentación complementaria}

En cuanto a la alimentación complementaria, en los tres territorios, los alimentos ofrecidos a los niños y las niñas menores de 2 años son similares. En Cartagena, sopas (de arroz, huevo), puré de papa, plátano, pasta, leguminosas; papillas, avena, arroz con leche, esporádicamente yogur. La mayoría de las bebidas son endulzadas con azúcar; inclusive, hubo un caso que mencionó la importancia de endulzar el tetero con una pizca de azúcar, que le preparaba al bebé para que mejorara la palatibilidad. También ofrecen jugos en caja, gaseosas y refrescos artificiales. Solo una de las participantes mencionó algunas frutas, y otra enunció que ofrece embutidos, como es el

‡ “[...] mecanismo mediante el cual la población más pobre del país, sin capacidad de pago, tiene acceso a los servicios de salud a través de un subsidio que ofrece el Estado" [24]. 
Tabla 3. Principales hallazgos de la práctica de la lactancia materna, por territorio

\begin{tabular}{|c|c|c|c|}
\hline Variable & Cartagena & Barranquilla & Sincelejo \\
\hline Exclusividad y beneficios & $\begin{array}{l}\text { - Introducción temprana de otros } \\
\text { alimentos } \\
\text { - Beneficios nutricionales }\end{array}$ & \multicolumn{2}{|c|}{$\begin{array}{l}\text { - Introducción temprana de otros alimentos } \\
\text { - Beneficios nutricionales y emocionales }\end{array}$} \\
\hline Prácticas y creencias & $\begin{array}{l}\text { - Uso de brasier roto } \\
\text { - Jeringa para sacar leche } \\
\text { - Para aumentar la producción: } \\
\text { alimentar a la madre con ajonjolí, } \\
\text { horchata y panela }\end{array}$ & $\begin{array}{l}\text { La leche materna } \\
\text { puede secarse; } \\
\text { por ello se debe } \\
\text { introducir otros } \\
\text { alimentos }\end{array}$ & $\begin{array}{l}\text { Para aumentar la } \\
\text { producción: tomar } \\
\text { líquidos, leche con panela, } \\
\text { ajonjolí y poner a mamar } \\
\text { un gato }\end{array}$ \\
\hline
\end{tabular}

salchichón, y en solo un caso se mencionó que ofrecía Bienestarina $^{\circledR}{ }^{\S}$

Allá me dijeron que le pusiera el [un alimento lácteo] a los 6 meses, cuando estaba allá hospitalizado. Después comencé con siete granos, azúcar (Entrevista madre lactante M, Cartagena).

Que comprara siete granos mientras tanto, y ahí le compré, y eso era lo que comía, hasta cuando tenía como 5 o 6 meses; yo empecé a darle puré de papa, plátano, sopita y todo, y ahora come de todo, de todo lo que le den, él se lo come (Grupo Focal, Cartagena).

En Barranquilla, señalaron que en la introducción de alimentos incluyen sopa de verduras, puré de verduras y de papa, agua, avena, huevo, pollo, arroz, pescado, carne, jugos, yogurt, compotas, plátano guineo, galleta, gelatina, ahuyama, pastas, yuca en puré, arepas de maíz, queso y pan. También mencionaron el uso de condimentos, como colorantes y potencializadores de sabor artificial, en las comidas.

[...] ya después de los 6 meses. Yo le empecé a dar por lo menos la sopita de verdura. No, darle sopita de verduras, pero este no. Eso él anda [...] A veces su mamá [ella se refiere de este modo a sí misma] se tomaba como dos o tres cucharitas porque no... Entonces no, apenas está empezando a tocar el... O sea, ellos todavía no sienten con el paladar, o sea, distinguir tantas cosas. Bueno, así ya después se le hacía puré.

$[\ldots]$

¿Qué otras cositas? O sea, cosas sencillitas, agüitas de cosas que no fueran a hacerle tanto daño. Ya después de los 9 meses, sí, eso empezó a darle de todo (Madre lactante A, Barranquilla, 2020).

En Sincelejo, los alimentos que generalmente ofrecen a los niños y las niñas son coladas de siete cereales, de arroz, sopa de verduras, papa, huevo, fruta, pollo, carne, galletas, pescado, Bienestarina ${ }^{\circledR}$, agua y aromática de manzanilla.

Por su parte, los alimentos considerados saludables en Cartagena son frutas, verduras y leche; en Barranqui1la, purés, plátano, verdura, fruta, panela, papa y pollo, mientras que en Sincelejo, las mujeres no aportaron elementos sobre este tema. Por otro lado, las entrevistadas refirieron que los alimentos no saludables son aquellos artificiales, como jugos de caja o refrescos en polvo.

En términos de alimentación perceptiva, ${ }^{* *}$ en los tres territorios afirman que les tienen sus propios utensilios para la alimentación de los niños y las niñas, como el plato, su cuchara, taza y vaso. Todas manifiestan que comparten el momento de alimentación con el niño o la niña, sin otra distracción. Solo en un caso dijo que le ofrece los alimentos en la moto de juguete. Requiere subir al niño a la moto, para que le reciba el alimento.

[...] De él, una cucharita pequeña, y su platico... y un vasito de él, mas nada (Madre lactante J, Sincelejo).

\section{Redes sociales que fortalecen el ejercicio de la maternidad y el cuidado de los niños y las niñas}

En los tres territorios, las madres mencionaron que para recibir asesoría respecto a la alimentación o al cuidado de los niños acudían con la suegra, la madre o la cuñada, mas no contemplan la institucionalidad como una fuente inmediata de apoyo para resolver estas inquietudes. En Sincelejo, las madres reconocen como una red de apoyo

\footnotetext{
§ "Es un alimento pre-cocido a base de una mezcla de cereales, leguminosa y leche entera en polvo, con vitaminas y minerales y ácidos grasos esenciales y de fácil preparación. Contiene micronutrientes aminoquelados que mejoran la absorción en la población beneficiaria y cuenta con un balance adecuado de aminoácidos esenciales. No contiene conservantes ni colorantes" [25].

** "Es una dimensión de la crianza perceptiva que implica reciprocidad entre el niño y su cuidador durante el proceso de alimentación” [26]
} 
efectiva las madres FAMI (madres del programa "Familia, Mujer e Infancia", del Instituto Colombiano de Bienestar Familiar),${ }^{\dagger \dagger}$ de las cuales algunas son beneficiarias.

Es importante anotar que, en Barranquilla, se evidenció una mayor participación del padre de los niños y las niñas en cuanto a apoyo económico, y también afectivo durante su cuidado.

Él dice que le tengo que dar la leche, dársela al bebé, porque a él también Clarita le explicó lo importante que era para el bebé, y él me decía que le tengo que dar y así [...] Él, en la mañana, me levanta y me dice: "Bueno. Vaya para donde Clarita, para que le des la leche al bebé". Yo me siento bien y él creo que también, porque él me dice que venga y eso (Madre lactante N, Barranquilla, 2020).

[...] que es muy importante que yo le dé la leche materna [...] Mi mamá me dice que lo mejor para el bebé es la leche del seno y todo eso [...] Que esa leche lo pone bonito, que lo fortalece, que lo hace crecer más rápido (Madre lactante $\mathrm{G}$, Barranquilla, 2020).

\section{Discusión}

Los resultados del análisis cualitativo evidenciaron que las madres gestantes y lactantes, beneficiarias de instituciones en procesos de implementación de la IAMII en los tres municipios, reconocieron los beneficios de la lactancia materna, desde el ámbito nutricional y emocional. Sin embargo, se identificó un abandono de la lactancia materna exclusiva, lo que puede deberse a que gran parte de las entrevistadas eran jóvenes y cuentan con poco acceso a la información.

Lo anterior está en concordancia con un estudio en Caldas, Colombia, que encontró que las madres reconocen los beneficios que ofrece la lactancia materna tanto en ellas como en sus bebés; no obstante, la exclusividad de la práctica no se cumple, tanto por la inexperiencia, que genera inseguridad en la madre, como también por la falta de apoyo familiar y comunitario [28].

Del mismo modo, la baja lactancia materna exclusiva se asocia con una alta tasa de embarazos adolescentes [29], lo que está directamente relacionado con la educación prenatal, puesto que a mayor educación, este fenómeno se convierte en un factor protector para la práctica de la lactancia materna [30]. Un estudio en Barranquilla encontró que quienes más se acercaron al tiempo ideal de lactancia materna exclusiva fueron las madres que recibieron capacitación y charlas antes y durante el embarazo [31].
En Turquía, un estudio que tuvo por objetivo determinar el tiempo, la tasa de la lactancia materna exclusiva y los factores que la afectan en madres adolescentes, encontró que las mujeres que iniciaron la lactancia materna habían planeado sus embarazos y recibieron educación en dicho tema [32].

En Galicia, España, una investigación determinó el nivel de conocimientos sobre lactancia materna y la influencia en la intención de amamantar después de la introducción de alimentación infantil a las 6 y 16 semanas de gestación y a los 6 meses posparto. Este estudio reportó que el 90,4 \% de las gestantes deseaban amamantar de manera exclusiva a su bebé; sin embargo, solo el 28,2 \% continuó hasta los 6 meses. En suma, el nivel de conocimientos en lactancia fue regular, tanto en la intención como en el tipo de alimentación en el recién nacido [33].

Estas situaciones descritas dan cuenta de la necesidad de generar estrategias de educación prenatal, como es también el caso, en Ahvaz, Irán, de un estudio que evaluó el efecto de un programa educativo sobre la autoeficacia y la duración de la lactancia materna, identificando que esta fue mayor en el grupo intervenido versus el grupo control (123,6 frente a 101,7, $p<0,001, \mathrm{y} 5,03$ meses vs. 2,7 meses, $p<0,001$, respectivamente) [34].

Un aspecto positivo es que, en Barranquilla, las mujeres valoraron el soporte emocional y económico que pueden brindarles sus compañeros. Un estudio en este municipio destacó que las participantes estimaron importante la aceptación de su estado de gestación en sus familias, amigos y allegados, sobre todo por el padre de su hijo [35]. Una investigación en Indiana, Estados Unidos, evaluó la participación del padre, reportando que el 45,9 \% ( $n$ : 67) de las madres recibieron ayuda de su esposo o pareja, mientras estaban el hospital, las cuales presentaron más probabilidades de continuar amamantando después de salir de este [36].

De igual forma, las madres entrevistadas identificaron la importancia de ofrecer huevo, pollo, pescado, frutas, algunos cereales y sus derivados a sus hijos menores de 2 años, lo cual se encuentra alineado a las últimas guías alimentarias en Colombia [37]. Sin embargo, reconocieron la introducción temprana de alimentos, lo que no se halla en línea con la recomendación de la oms de ofrecer lactancia materna exclusiva hasta los 6 meses [38].

Cabe anotar que los diferentes estudios refieren que la lactancia materna ofrece beneficios a corto y a largo plazo en los niños y las niñas, como la mejora en el desarrollo cognitivo, bajas tasas de obesidad, disminución del riesgo de diabetes, hipertensión, enfermedad

$\dagger$ "Es un servicio de atención, diseñado para apoyar grupos entre doce (12) y quince (15) familias en desarrollo en el mejoramiento de las relaciones intrafamiliares y el fortalecimiento de vínculos afectivos, para que apoyen el desarrollo de los niños y niñas desde su gestación, vinculando además a otros adultos para que participen de la crianza de los niños" [27]. 
cardiovascular e hiperlipidemia, entre otras [39]. En las madres, ayuda a que el útero regrese a su tamaño previo, reduce el sangrando y previene la anemia; disminuye el riesgo de padecer cáncer de ovario y mamá, genera una relación cercana y de amor entre la madre y el bebé, entre otros beneficios [40].

De acuerdo con Lancet, no amamantar se asocia con una menor inteligencia y pérdidas económicas de alrededor de \$302 mil millones de dólares anuales o $0,49 \%$ del ingreso bruto mundial, debido a que la lactancia materna proporciona ventajas de salud, económicas y ambientales a corto y largo plazo para los niños, las mujeres y la sociedad [41].

Por todo ello, es necesario seguir fortaleciendo esta práctica hasta los 6 meses de manera exclusiva y con alimentación complementaria hasta los 2 años o más, teniendo en cuenta que en el territorio interés del estudio la prevalencia de la práctica es de 20,5\% [16].

En concordancia con la alimentación complementaria, las entrevistadas refirieron también brindar alimentos de consistencia líquida, como sopas, contrario a la orientación de iniciar con alimentos espesos e ir progresando paulatinamente a consistencias más sólidas. A su vez, identificaron incluir alimentos con alto contenido de azúcar, condimentos y jugos de caja, lo cual no hace parte de una alimentación saludable [37].

De igual forma, se resalta que, en general, las entrevistadas mencionaron que sus hijos contaban con sus propios utensilios de cocina, como plato, vaso y cuchara, para consumir alimentos. Asimismo, refirieron destinar tiempo para ofrecer la alimentación a sus hijos. Lo anterior da cuenta de la reciprocidad entre el niño y el cuidador durante el proceso de alimentación, que corresponde finalmente al concepto de alimentación percepti$v a[42,43]$. Esta última enfatiza en la importancia de que los cuidadores decidan cuándo empezar y cuándo dejar de alimentar al niño, con base en sus señales de hambre y saciedad [26].

Por otro lado, en general, las madres gestantes y lactantes también reconocieron la importancia de contar con el apoyo de programas en modalidad FAMI; no obstante, una investigación en Cartagena reveló que si bien en muchas oportunidades son el primer contacto con la madre, requieren de más actualización y formación en lactancia materna [44]. En Murcia, España, un estudio identificó el impacto de las redes sociales sobre la lactancia materna, las cuales tienen una influencia positiva en la duración de la decisión de la mujer de amamantar [45]. Por lo tanto, es necesario visibilizar las instituciones y redes que contribuyen con el fomento de la práctica.

Teniendo en cuenta lo anterior, esta investigación permitió identificar que las prácticas de alimentación infantil en instituciones de salud priorizadas que se encuentran en el proceso de implementación de la IAMII, presentan avances en el ámbito de la alimentación infantil, puesto que las madres refirieron conocer los beneficios de la lactancia materna y mencionaron elementos de apropiación en alimentación perceptiva; además, reconocen redes de apoyo para el proceso de alimentación del lactante y el niño pequeño. Sin embargo, aún existen factores que limitan las prácticas adecuadas de alimentación infantil, como lo son algunas creencias, que deben ser estudiadas y mediadas, teniendo en cuenta el contexto cultural.

En síntesis, esta investigación permitió reconocer los factores que favorecen y limitan las adecuadas prácticas de alimentación del lactante y del niño pequeño, en madres gestantes y lactantes, y su red de apoyo, que viven en condiciones de vulnerabilidad social y económica, $\mathrm{y}$ son atendidas en tres instituciones de salud del caribe colombiano que se encuentran en proceso de implementación de la estrategia IAMII. Esta evidencia posibilita avanzar en la formulación de procesos de educación en salud, orientados a los contextos locales, no solo a las instituciones que participaron del estudio, sino también a otras instituciones y territorios que estén en proceso de implementación de la IAMII. Asimismo, permite formular otras expresiones de política en torno a la alimentación infantil, como lo son la socialización de las "Guías alimentarias basadas en alimentos para mujeres gestantes, madres en período de lactancia y niños y niñas menores de dos años para Colombia" [37], la Resolución 3280 de 2018 [46], el Plan Decenal de Salud Pública [47], el Plan Decenal de Lactancia materna [48], y la Política de Seguridad Alimentaria y Nutricional [49].

\section{Limitaciones}

Los resultados de este estudio no pueden ser generalizados a otras instituciones de salud o a la totalidad de la población de Cartagena, Barranquilla y Sincelejo, puesto que la muestra definida fue por conveniencia. Por lo tanto, para mayores aproximaciones al fenómeno, deben contemplarse estudios que vinculen otras instituciones de salud y otros métodos de investigación.

\section{Agradecimientos}

Agradecemos a territorios, Secretarías de Salud e instituciones de salud participantes: Clínica General del Caribe, Camino Bosque de María e IPs San Luis. De igual forma, al Instituto Colombiano de Bienestar Familiar, líderes comunitarios y de manera muy especial a las gestantes y lactantes que decidieron participar en el proceso de investigación. 


\section{Declaración de fuente de financiación}

Fundación Éxito y Fundación Santa Fe de Bogotá.

\section{Declaración de conflictos de intereses}

Las autoras declaramos no tener ningún conflicto de interés.

\section{Declaración de responsabilidad}

Las autoras certificamos que participamos suficientemente del trabajo para tornar pública nuestra de la responsabilidad por el contenido; certificamos que el artículo representa un trabajo original y que ni este manuscrito, en parte o en su totalidad, ni otro trabajo con contenido sustancialmente similar, de nuestra autoría, fue publicado o está siendo considerado para publicación en otra revista, sea en formato impreso o electrónico. También damos fe de que si fuese solicitado, daremos o cooperaremos en la obtención y entrega de datos sobre los cuales el artículo está basado, para examen de quien lo requiera.

\section{Declaración de contribución por autores}

Nancy Millán fue quien lideró la concepción, el diseño e interpretación de datos, la construcción y la revisión final del artículo.

Paula Castro y Sonia Fernández apoyaron la concepción, la recolección de información, la construcción y la revisión final del manuscrito.

Gina Arocha apoyó la revisión de la información y la revisión final del documento.

\section{Referencias}

1. Ministerio de Salud y Protección Social, Organización Panamericana de la Salud y Organización Mundial de la Salud. Fortalecimiento de la estrategia IAMI integral en departamentos priorizados. Convenio 519 de 2015. Anexo 2: lineamientos para la implementación y fortalecimiento de la estrategia IAMII 2016.

2. Nobari TZ, Jiang L, et al. Baby-Friendly Hospital Initiative and Breastfeeding Among wIC-participating infants in Los Angeles county. J Hum Lact. 2017;33(4):677-83. Dor: https://doi. org $/ 10.1177 / 0890334417716118$

3. Munn AC, Newman SD, Mueller M, et al. The Impact in the United States of the Baby-Friendly Hospital Initiative on early infant health and breastfeeding outcomes. Breastfeed Med. 2016;11(5):222-30. DoI: https://doi.org/10.1089/bfm.2015.0135

4. Guedes Araujo R, Matos Fonseca V de, et al. External evaluation and self-monitoring of the Baby-friendly Hospital Initiative's maternity hospitals in Brazil. Int Breastfeed J. 2019;14,1. DoI: https:// doi.org/10.1186/s13006-018-0195-4
5. Cabrera G. La iniciativa de institución amiga de la mujer y la infancia (IAMI) en hospitales del Valle del Cauca. Rev. Fac. Nac. Salud Pública [internet]. 2002 [citado 2020 sep. 9]; 20(1):75-88. Disponible en: https://www.redalyc.org/pdf/120/12020107.pdf

6. López SR, Díaz MC, Hernández BN, Mercado ML, Aguilar AC, Cifuentes SL. Percepción de la iniciativa Instituciones Amigas de la Mujer y la Infancia (IAMI). Rev Cienc Salud [internet]. 2015 [citado 2020 sep. 9]; 13(3):395-409. Disponible en: https://revistas. urosario.edu.co/xml/562/56242524007/index.html

7. Organización Mundial de la Salud. El hambre en el mundo sigue aumentando, advierte un nuevo informe de la onU [internet]; 2018 [citado 2020 sep. 9]. Disponible en: https://www.who.int/es/newsroom/detail/11-09-2018-global-hunger-continues-to-rise---newun-report-says

8. Instituto Nacional de Estadística y Geografía, Instituto Nacional de Salud Pública, Secretaría de Salud. Encuesta Nacional de Salud y Nutrición 2018 [internet]; 2018 [citado 2020 sep. 9]. Disponible en: https://ensanut.insp.mx/encuestas/ensanut2018/doctos/informes/ensanut_2018_presentacion_resultados.pdf

9. Organización Panamericana de la Salud. Los bebés y las madres del mundo sufren los efectos de la falta de inversión en la lactancia materna [internet]; 2017 [citado 2020 sep. 9]. Disponible en: https://www.paho.org/per/index.php?option=com content \&view $=$ article $\&$ id $=3850: 1$ sos-bebes-y-las-madresdel-mundo-sufren-los-efectos-de-la-falta-de-inversion-en-lalactancia-materna\&Itemid=900\#: :text $=\mathrm{Seg} \% \mathrm{C} 3 \% \mathrm{BAn} \% 20$ la $\% 20$ informaci $\%$ C3\%B3n\%20proporcionada $\% 20$ por,el $\% 20$ Per\%C3\%BA\%20es\%20del\%2069.8\%25

10. Estado Plurinacional de Boivia. Ministerio de Salud y Deportes. Bolivia es el segundo país con mayor índice de lactancia materna exclusiva [internet]; 2015 [citado 2020 sep. 9]. Disponible en: https://www.minsalud.gob.bo/467-bolivia-es-el-segundo-paiscon-mayor-indice-de-lactancia-materna-exclusiva

11. Argentina, Ministerio de Salud de la Nación, Dirección Nacional de Maternidad, Infancia y Adolescencia. Situación de la lactancia materna en la Argentina [internet]; 2015 [citado 2020 sep. 9]. Disponible en: https://bancos.salud.gob.ar/sites/default/files/201810/0000001135cnt-lactancia-materna-argentina-2015.pdf

12. Pantoja-Mendoza IY, Meléndez G, et al. Review of complementary feeding practices in Mexican children. Nutr Hosp. 2015;31(2):552-8. DoI: https://doi.org/10.3305/nh.2015.31.2.7668

13. Rojas DC, Ysla M, Riega DV, et al. Enfermedades diarreicas, infecciones respiratorias y características de la alimentación de los niños de 12 a 35 meses de edad en El Perú. Rev Peru Med Exp Salud Pública [internet]. 2004 [citado 2020 sep. 9]; 21(3):14656. Disponible en: http://www.scielo.org.pe/pdf/rins/v21n3/ a06v21n3.pdf

14. Abeldaño RA, López NM, Burrone MS, et al. Prácticas de lactancia y alimentación complementaria en menores de 6 meses en Argentina. Estimaciones a partir de una encuenta multipropósito. Revista de Salud Pública [internet]. 2015 [citado 2020 sep. 9]; 19(1):50-8. Disponible en: http://www.saludpublica.fcm.unc.edu. ar/sites/default/files/RSP15_1_07_\%20art4.pdf

15. Cruz AY, Jones AD, et al. Lactancia materna, alimentación complementaria y malnutrición infantil en los Andes de Bolivia. ALAN [internet]. 2010 [citado 2020 sep. 9]; 60(1):7-14. Disponible en: http://ve.scielo.org/scielo.php?script=sci_arttext\&pid=S000406222010000100002\&lng=es

16. Universidad Nacional de Colombia, Instituto Nacional de Salud, Instituto Colombiano de Bienestar Familiar. Encuesta Nacional de la Situación Nutricional. Bogotá: Gobierno de Colombia; 2015. 
17. Cordón SE. Módulo propedéutico: introducción a la investigación. Bilwi: Universidad de las Regiones Autónomas de la Costa Caribe Nicaraguense (URACCAN), Maestría en salud intercultural con mención en salud sexual y reproductiva; 2010.

18. Sandoval C. Investigación cualitativa. Enfoques y modalidades de investigación cualitativa: rasgos básicos. En M. Duriez González, Curso investigación cualitativa y mixta. Managua: URACCAN; 2008.

19. Bonilla E, Rodríguez P. Más allá del dilema los métodos. Bogotá: Norma. Universidad de los Andes; 1997.

20. Stracuzzi SP, Martins FP. Metodología de la investigación cuantitativa. Caracas: Fedupel; 2012.

21. Habegger S, Mancila I. El poder de la cartografía social en las prácticas contrahegemónicas o la cartografía social como estrategia para diagnosticar nuestro territorio [internet]; 2006 [citado 2020 sep. 9]. Disponible en: http://beu.extension.unicen.edu. ar/xmlui/bitstream/handle/123456789/365/Habegger\%20y\%20 Mancila_El\%20poder\%20de \%201a\%20cartografia\%20social. pdf? sequence $=1$ \&isAllowed $=\mathrm{y}$

22. Hamui SA, Varela RM. La técnica de grupos focales. Investigación en Educación Médica [internet]. 2013 [citado 2020 sep. 9]; 2(1):55-60. Disponible en: https://www.redalyc.org/articulo. oa? id=349733230009

23. Ministerio de Salud y Protección Social. Resolución 008430 de 1993, por la cual se establecen las normas científicas, técnicas y administrativas para la investigación en salud (1993 oct. 4) [internet]; 1993 [citado 2020 sep. 9]. Disponible en: https://www. hospitalsanpedro.org/images/Comite_Investigacion/Resolucion_8430_de_1993.pdf

24. Colombia, Ministerio de Salud y Protección Social. Régimen subsidiado [internet]. s. f. [citado 2020 sep. 9]. Disponible en: https://www.minsalud.gov.co/salud/ Paginas/R\%C3\%A9gimenSubsidiado.aspx\#: :text=E1\%20 R\%C3\%A9gimen\%20Subsidiado\%20es\%20el,subsidio\%20 que $\% 20$ ofrece $\% 20$ el $\% 20$ Estado

25. Instituto Colombiano de Bienestar Familiar. Bienestarina Más $₫$ [internet]. s. f. [citado 2020 sep. 9]. Disponible en: https://www. icbf.gov.co/bienestarina-mas-r-0

26. Pérez ER, Segura PS, Lott M. Guías de alimentación para niñas y niños menores de dos años: un enfoque de crianza perceptiva. Investigación en Alimentación Saludable. Prevención de obesidad infantil basada en evidencia. Febrero; 2017.

27. Instituto Colombiano de Bienestar Familiar. Familia Mujer e Infancia FAMI [internet]. s. f. [citado 2020 sep. 9]. Disponible en: https://www.icbf.gov.co/portafolio-de-servicios-icbf/familia-mujer-e-infancia-fami

28. Benjumea RM, Falla M, Jurado VL. Conocimientos y prácticas de gestantes y cuidadores sobre lactancia materna en cinco municipios caldenses. Colombia. Hacia Promoc Salud [internet]. 2013 [citado 2020 sep. 9]; 18(2):66-78. Disponible en: http://www.scielo.org.co/pdf/hpsal/v18n2/v18n2a06.pdf

29. López SR, P. Ángel P, et al. Práctica de lactancia materna en adolescentes en Cartagena [Ponencia]. Memorias del Encuentro Internacional de Investigadores en Lactancia Materna. Universidad de Cartagena, Cartagena de Indias [internet]; 2018 [citado 2020 sep. 9]. Disponible en: http://observatorio.saluddecaldas.gov.co/desca/ san/revista\%20lactancia\%20Cartagena.pdf

30. Roig OA, Martínez MR, et al. Factors associated to breastfeeding cessation before 6 months. Rev Latino-Am. Enfermagem. 2010;18(3):373-80. DoI: https://doi.org/10.1590/S010411692010000300012
31. Bustamante VM, Ramos SS. Factores asociados a las prácticas de la lactancia materna en una población de niños sanos nacidos en el Hospital Niño Jesús, periodo 2017-2018 [trabajo de especialista], [Barranquilla]: Universidad Simón Bolívar [internet]; 2018 [citado 2020 sep. 9]. Disponible en: https://bonga.unisimon.edu.co/ handle/20.500.12442/3339

32. Yılmaz E, Yılmaz Z, Isık H, et al. Factors associated with breastfeeding initiation and exclusive breastfeeding rates in turkish adolescent mothers. Breastfeed Med. 2016;11(6):315-20. Dor: https:// doi.org/10.1089/bfm.2016.0012

33. Suárez-Cotelo MDC, Movilla-Fernández MJ, Pita-García P, Arias BF, Novío S. Breastfeeding knowledge and relation to prevalence. Rev Esc Enferm USP. 2019;53:1-9. DoI: https://doi.org/10.1590/ S1980-220X2018004503433

34. Ansari S, Abedi P, Hasanpoor S, Bani S. The effect of interventional program on Breastfeeding self-efficacy and duration of exclusive breastfeeding in pregnant women in Ahvaz, Iran. Int Sch Res Notices. 2014:510793. DoI: https://doi.org/10.1155/2014/510793

35. Muñoz HM, Pardo TM. Significado de las prácticas de cuidado cultural en gestantes adolescentes de Barranquilla (Colombia). Aquichan. 2016;16(1):43-55. Dor: https://doi.org/10.5294/ aqui.2016.16.1.6

36. Hunter T, Cattelona G. Breastfeeding initiation and duration in first-time mothers: Exploring the impact of father involvement in the early post-partum period. Health Promot Perspect. 2014;4(2):132-6. DOI: https://doi.org/10.5681/hpp.2014.017

37. Instituto Colombiano de Bienestar Familiar, Organización de las Naciones Unidas para la Alimentación y la Agricultura. Guías alimentarias basadas en alimentos para mujeres gestantes, madres en período de lactancia y niños y niñas menores de dos años para Colombia. Bogotá; 2018.

38. Organización Mundial de la Salud. Lactancia materna exclusiva [internet]; 2020 [citado 2020 jun. 3]. Disponible en: https://www. who.int/elena/titles/exclusive_breastfeeding/es/

39. Binns C, Lee M, Low WY. The long-term public health benefits of breastfeeding. Asia Pac J Public Health. 2016;28(1):7-14. DoI: https://doi.org/10.1177/1010539515624964

40. Organización Mundial de la Salud, Organización Panamericana de la Salud, Unicef. Consejería para la Alimentación del Lactante y Niño Pequeño: Curso Integrado. Suiza [internet]; 2009 [citado 2020 sep. 9]. Disponible en: https://apps.who.int/iris/bitstream/ handle $/ 10665 / 44047 / 9789243594767$ spa.pdf;jsessionid=4A5EE C52185DAA96A711D48D1C132319? sequence $=3$

41. Rollins NC, Bhandari N, Hajeebhoy N, et al. Why invest, and what it will take to improve breastfeeding practices? Lancet. 2016;387(10017):491-504. DoI: https://doi.org/10.1016/S01406736(15)01044-2

42. Bentley ME, Wasser HM, Creed KH. Responsive feeding and child undernutrition in low- and middle-income countries. J Nutr. 2011;141(3):502-7. DoI: https://doi.org/10.3945/jn.110.130005

43. DiSantis KI, Hodges EA, et al. The role of responsive feeding in overweight during infancy and toddlerhood: A systematic review. Int J Obes (Lond). 2011;35(4):480-92. DOI: https://doi. org/10.1038/ijo.2011.3

44. Bueno Y. Conocimiento sobre lactancia materna en gestoras educativas "Madres FAMI" del área rural y urbana de una fundación de Cartagena [tesis de grado]. [Cartagena de Indias]: Universidad de Cartagena, Facultad de Enfermería [internet]; 2018 [citado 2020 jun. 4]. Disponible en: https://repositorio.unicartagena. edu.co/bitstream/handle/11227/7006/CONOCIMIENTO $\% 20$ SOBRE\%20LACTANCIA\%20MATERNA\%20EN\%20GESTO- 
RAS\%20EDUCATIVAS 1.pdf;jsessionid=90CF7EF6D1B17918 30588D6F0D31B787? sequence $=1$

45. Baño PI, Martínez RM, Canteras JM, et al. Impact of support networks for breastfeeding: A multicentre study. Women Birth. 2018;31(4):e239-e244. DoI: https://doi.org/10.1016/j. wombi.2017.10.002

46. Colombia, Ministerio de Salud y Protección Social. Resolución 3280 de 2018 [internet]; 2018 [citado 2021 ene. 21]. Disponible en: https://www.minsalud.gov.co/sites/rid/Lists/BibliotecaDigital/RIDE/DE/DIJ/resolucion-3280-de-2018.pdf

47. Colombia, Ministerio de Salud y Protección Social. ABC del Plan Decenal de Salud Pública [internet]; 2013 [citado 2021 ene. 21].
Disponible en: https://www.minsalud.gov.co/sites/rid/Lists/BibliotecaDigital/RIDE/VS/ED/PSP/IMP_4feb+ABCminsalud.pdf

48. Colombia, Ministerio de la Protección Social. Plan Decenal Lactancia materna [internet]; 2010 [citado 2021 ene. 21]. Disponible en: https://www.minsalud.gov.co/sites/rid/Lists/BibliotecaDigital/RIDE/VS/PP/SNA/plan-decenal-lactancia-materna.pdf

49. Colombia, Consejo Nacional de Política Económica Social, Departamento Nacional de Planeación. Política Nacional de Seguridad Alimentaria y Nutricional [internet]; 2010 [citado 2021 ene. 21]. Disponible en: https://www.siteal.iiep.unesco.org/sites/ default/files/sit_accion_files/co_0442.pdf 